\title{
CHEMICAL MODIFICATIONS OF 6-ALLYLSULFONYL- 4-METHYL-1,2-DIHYDROQUINOLIN-2-ONE AS THE APPROACH FOR SEACHING NEW BIOLOGICALLY ACTIVE SUBSTANCES
}

\author{
T.O.Tsapko \\ National University of Pharmacy \\ Key words: 1,2-dihydroquinoline-2-one; allylsulfone; heterocyclic sulfones; nucleophilic addition
}

To search biologically active substances among quinolone-2 sulfonyl derivatives the method for obtaining of the convenient intermediate, namely 6-allylsulfonyl-4-methyl-1,2-dihydroquinoline-2-one, has been developed. The synthesis has been carried out by alkylation of the initial 4-methyl-2-oxo1,2-dihydroquinoline-6-sulfinic acid with allyl bromide in the acetonitrile medium and in the presence of potassium carbonate giving $73 \%$ yield of the product. The presence of the allyl fragment of 6-allylsulfonyl-4-methyl-1,2-dihydroquinoline-2-one allowed to carry out addition reactions with some $\mathrm{C}$-, $\mathrm{N}$ - and O-nucleophiles. Addition to allyl sulfones occurs in accordance with Markovnikov's rule despite of the strong electron withdrawing effect of the sulfonyl group. The reaction of 6-allylsulfonyl4-methyl-1,2-dihydroquinoline-2-one with active methylene compounds has been carried out with diethyl malonate and ethyl cyanoacetate by refluxing in absolute ethanol in the presence of sodium ethoxide. As a result, 2-R-3-methyl-4-(4-methyl-2-oxo-1,2-dihydroquinoline-6-sulfonyl)butanoic acid ethyl esters have been obtained with 73-79\% yields. 6-(2-Alkylaminopropylsulfonyl)-4-methyl-1,2dihydroquinoline-2-ones are formed under the action of $\mathrm{N}$-nucleophilic reagents, in particular primary aliphatic amines, on 6-allylsulfonyl-4-methyl-1,2-dihydroquinoline-2-one. This reaction takes place upon heating in dimethylformamide up to $50-60^{\circ} \mathrm{C}$ for 6 hours, the yields of the products are 35-40\%. Hydration of the allyl fragment occurs in the same solvent when treated with sodium hydroxide at $20^{\circ} \mathrm{C}$ resulting in formation of 6-(2-hydroxypropylsulfonyl)-4-methyl-1,2-dihydroquinolin-2-one with 62\% yield. The chemical transformations described open a prospect for synthesis of variety of new compounds with different functional groups in the alkylsulfonyl moiety of quinolones. This approach also allows to influence on such properties of new biologically active substances as molecular weight, lipophilicity, acidity, etc., which are essential for permeability of substances through biological membranes.

According to the recent literature data, most of the studies is devoted to aryl sulfones, while heteryl sulfones have not been investigated so intensively. Nevetheless, some compounds of this class with the antimicrobial [8], antiviral [9, 11], antimalarial [5], anti-inflammatory and analgesic $[10,12,13]$, antitumor [6] action have already been found. Therefore, sulfones is a promising class for study in order to develop medicinal substances. Continuing the research of 1,2-dihydroquinoline-2-one derivatives [2] that are carried out in the National University of Pharmacy a number of new potential biologically active substances in the range of 6-sulfonyl derivatives of 4-methyl-1,2-dihydroquinoline-2-one have been obtained by the reaction of nucleophilic addition to 6-allylsulfonyl-4-methyl-1,2-dihydroquinoline-2-one.

\section{Materials and Methods}

Methods of organic synthesis were used for obtaining new compounds. The structures of the compounds synthesized were proven by the methods of ${ }^{1} \mathrm{H}$ NMR spectroscopy and mass spectrometry. ${ }^{1} \mathrm{H}$ NMR spectra were recorded on a Varian Mercury VX-200 in DMSO$\mathrm{D}_{6}$ solution, the operating frequency was $200 \mathrm{MHz}$, the internal standard - TMS. Mass spectra were recorded on a Varian $1200 \mathrm{~L}$, the ionizing voltage was $70 \mathrm{eV}$. Melting points were measured by a Koffler device.

\section{Results and Discussion}

The method of synthesis of 6-allylsulfonyl-4-methyl1,2-dihydroquinolin-2-one (2) by alkylation of 4-methyl2-oxo-1,2-dihydroquinoline-6-sulfinic acid (1) with allyl bromide has been developed (Scheme 1). The reaction was carried out in acetonitrile medium in the presence of potassium carbonate for $2 \mathrm{~h}$. The product 2 was obtained with the yield of $73 \%$ [1].

Compound 2 was used for further chemical modifications due to the presence of the allyl fragment. It is known that despite of the strong electron withdrawing effect of the sulfonyl group addition of nucleophiles to allyl sulfones occurs in accordance with Markovnikov's rule [3]. And the products of this reaction are interesting objects of studying from the pharmacological point of view [4]. Therefore, to continue the search of biologically active compounds in the range of sulfonyl derivatives of 4-methyl-1,2-dihydroquinoline-2-one the addition reactions of some nucleophilic reagents to allyl sulfone 2 were carried out. Several active methylene compounds, alkylamines, as well as sodium hydroxide were selected for these reactions (Scheme).

The reactions of 6-allylsulfonyl-4-methyl-1,2-dihydroquinoline-2-one (2) with diethyl malonate and ethyl cyanoacetate in absolute ethanol in the presence of so- 


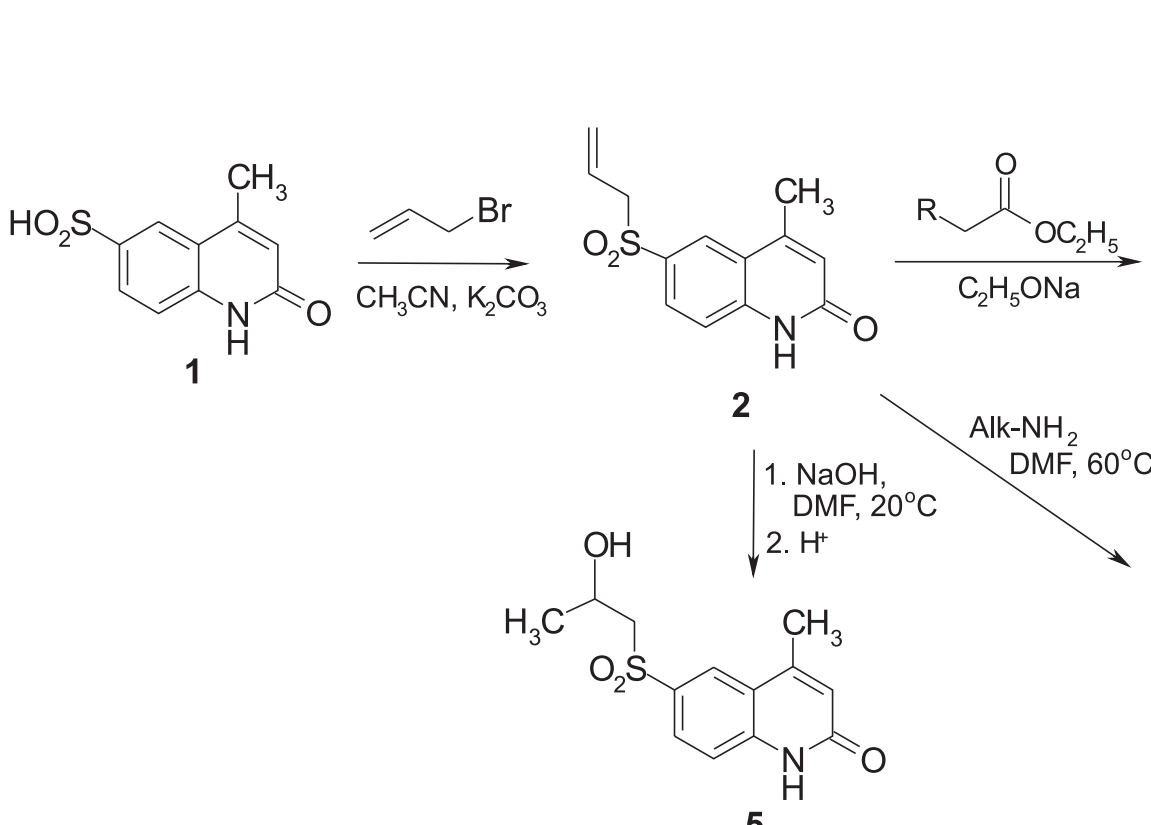<smiles>[R]C(C(=O)OCC)C(C)CS(=O)(=O)c1ccc2[nH]c(=O)cc(C)c2c1</smiles>

$3 a, b$ $\mathrm{R}=\mathrm{a}) \mathrm{COOC}_{2} \mathrm{H}_{5}$, b) $\mathrm{CN}$<smiles>Cc1cc(=O)[nH]c2ccc(S(=O)(=O)CC(C)N[AlH2])cc12</smiles>

$4 a, b$

Alk = a) $\mathrm{CH}_{2} \mathrm{C}_{6} \mathrm{H}_{5}$, b) $\mathrm{H}-\mathrm{C}_{6} \mathrm{H}_{13}$ dium ethoxide for $2 \mathrm{~h}$ under the reflux were performed. As a result, 2-R-3-methyl-4-(4-methyl-2-oxo-1,2-dihydroquinoline-6-sulfonyl)butanoic acid ethyl esters $(\mathbf{3 a}, \mathbf{b})$ were obtained with high yields (Tab. 1).

6-(2-Alkylaminopropylsulfonyl)-4-methyl-1,2-dihydroquinoline-2-ones $(\mathbf{4 a}, \mathbf{b})$ were synthesized by addition of the primary aliphatic amines to compound $\mathbf{2}$. The process was performed in DMFA for 6h when heating up to $50-60^{\circ} \mathrm{C}$. The compounds $\mathbf{4 a}$, $\mathbf{b}$ obtained can be considered as promising objects for studying their effects on energy processes in the body because of the fragment of 2-aminoethanesulfonic acid $\left(\mathrm{NH}_{2}-\left(\mathrm{CH}_{2}\right)_{2}-\mathrm{SO}_{3} \mathrm{H}\right)$ known as taurine that has diverse physiological effects [5].

As it was shown in our previous work [1], heating of allyl sulfone $\mathbf{2}$ in aqueous alkaline solutions caused cleavage of the C-S bond. And sodium salt of 4-methyl2-oxo-1,2-dihydroquinoline-6-sulfinic acid was obtained as the product. Thus, conditions of hydration of the double bond of 6-allylsulfonyl-4-methyl-1,2-dihydroquinoline2 -one (2) were investigated. It has been found that $\beta$-hydroxy derivative, namely 6-(2-hydroxy propylsulfonyl)4-methyl-1,2-dihydroquinolin-2-one (5) is formed when intermediate $\mathbf{2}$ is treated with sodium hydroxide in DMFA solution at $20^{\circ} \mathrm{C}$.

Compounds $\mathbf{3 a}, \mathbf{b}, \mathbf{4 a}, \mathbf{b}$ and $\mathbf{5}$ are white crystalline substances, soluble in polar organic solvents, insoluble in water. Data of ${ }^{1} \mathrm{H}$ NMR spectroscopy and mass spectrometry of the compounds obtained are presented in Tab. 2.

Synthesis of 2-ethoxycarbonyl-3-methyl-4-(4-methyl2-oxo-1,2-dihydroquinolin-6-sulfonyl)butanoic acid ethyl ester (3a). Boil 0.01 mole of diethyl malonate for $30 \mathrm{~min}$ in sodium ethoxide solution (prepared from $0.23 \mathrm{~g}$ of metallic sodium and $20 \mathrm{ml}$ of absolute ethanol). Add 0.01 mole $(2.63 \mathrm{~g})$ of allyl sulfone 2 and then boil for 2 hours. Evaporate the solvent under vacuum. Add $50 \mathrm{ml}$ of water, and acidify the mixture with hydrochloric acid to $\mathrm{pH}=5$. Filter the precipitate, crystallize with $50 \%$ ethanol.

2-Cyano-3-methyl-4-(4-methyl-2-oxo-1,2-dihydroquinoline-6-sulfonyl) butanoic acid ethyl esters (3b) was prepared similarly to compound 3a using ethyl cyanoacetate instead of diethyl malonate.

Synthesis of 6-(2-hexylaminopropylsulfonyl)-4-methyl-1,2-dihydroquinolin-2-one (4a). Heat 0.01 mole

Table 1

Properties of the compounds synthesized<smiles>[R]C(C)COc1ccc2[nH]c(=O)cc(C)c2c1</smiles>

\begin{tabular}{|c|c|c|c|c|}
\hline Compound & $\mathrm{R}$ & Formula & m.p., ${ }^{\circ} \mathrm{C}$ & Yield, \% \\
\hline $3 a$ & $\mathrm{CH}\left(\mathrm{COOC}_{2} \mathrm{H}_{5}\right)_{2}$ & $\mathrm{C}_{20} \mathrm{H}_{25} \mathrm{NO} \mathrm{O}_{7} \mathrm{~S}$ & $184-186$ & 79 \\
\hline $3 \mathrm{~b}$ & $\mathrm{CH}(\mathrm{CN}) \mathrm{COOC}_{2} \mathrm{H}_{5}$ & $\mathrm{C}_{18} \mathrm{H}_{20} \mathrm{~N}_{2} \mathrm{O}_{5} \mathrm{~S}$ & $197-198$ & 73 \\
\hline $4 a$ & $\mathrm{NH}-\left(n-\mathrm{C}_{6} \mathrm{H}_{13}\right)$ & $\mathrm{C}_{19} \mathrm{H}_{28} \mathrm{~N}_{2} \mathrm{O}_{3} \mathrm{~S}$ & $200-220$ & 35 \\
\hline $4 \mathrm{~b}$ & $\mathrm{NHCH} \mathrm{C}_{6} \mathrm{H}_{5}$ & $\mathrm{C}_{20} \mathrm{H}_{22} \mathrm{~N}_{2} \mathrm{O}_{3} \mathrm{~S}$ & $241-243$ & 40 \\
\hline 5 & $\mathrm{OH}$ & $\mathrm{C}_{13} \mathrm{H}_{15} \mathrm{NO}_{4} \mathrm{~S}$ & $198-200$ & 62 \\
\hline
\end{tabular}


${ }^{1} \mathrm{H}$ NMR spectroscopy and mass spectrometry data of the compounds synthesized

\begin{tabular}{|c|c|c|c|c|c|c|c|c|c|c|c|}
\hline \multirow{3}{*}{ 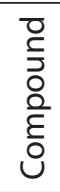 } & \multicolumn{10}{|c|}{${ }^{1} \mathrm{H}$ NMR spectrum, $\delta$, ppm } & \multirow{3}{*}[\mathrm{M}^{+}]{} \\
\hline & \multirow{2}{*}{$\begin{array}{r}1-\mathrm{NH} \\
(1 \mathrm{H}, \mathrm{s})\end{array}$} & \multicolumn{4}{|c|}{$\mathrm{H}_{\text {quin. }}$} & \multirow{2}{*}{$\begin{array}{l}4-\mathrm{CH}_{3} \\
(3 \mathrm{H}, \mathrm{s})\end{array}$} & \multirow{2}{*}{$\begin{array}{l}\mathrm{CH}_{2} \\
(2 \mathrm{H}, \mathrm{d})\end{array}$} & \multirow{2}{*}{$\begin{array}{c}\mathrm{CH} \\
(1 \mathrm{H}, \mathrm{m})\end{array}$} & \multirow{2}{*}{$\begin{array}{l}\mathrm{CH}_{3} \\
(3 \mathrm{H}, \mathrm{d})\end{array}$} & \multirow[b]{2}{*}{$\mathrm{R}$} & \\
\hline & & $\begin{array}{c}5-\mathrm{H} \\
(1 \mathrm{H}, \mathrm{d})\end{array}$ & $\begin{array}{c}7-\mathrm{H} \\
(1 \mathrm{H}, \mathrm{dd}) \\
\end{array}$ & $\begin{array}{c}8-\mathrm{H} \\
(1 \mathrm{H}, \mathrm{d})\end{array}$ & $\begin{array}{c}3-\mathrm{H} \\
(1 \mathrm{H}, \mathrm{s})\end{array}$ & & & & & & \\
\hline $3 a$ & 12.04 & 8.11 & 7.94 & 7.46 & 6.53 & 2.47 & 3.37 & $\begin{array}{l}2.50- \\
2.60\end{array}$ & $\begin{array}{l}1.08- \\
1.12^{*}\end{array}$ & $\begin{array}{l}4.05\left(4 \mathrm{H}, \mathrm{q}, 2 \mathrm{C}_{2} \mathrm{CH}_{3}\right) ; \\
\left.3.61\left(1 \mathrm{H}, \mathrm{d}, \mathrm{C} \underline{(\mathrm{COOCH}} \mathrm{CH}_{3}\right)_{2}\right) ; \\
1.08-1.12^{*}\end{array}$ & 423 \\
\hline $3 b$ & 12.03 & 8.16 & 7.96 & 7.47 & 6.54 & 2.48 & 3.45 & $\begin{array}{l}2.55- \\
2.61\end{array}$ & 1.22 & $\begin{array}{l}4.40(1 \mathrm{H}, \mathrm{d}, \mathrm{CHCN}) ; 4.15(2 \mathrm{H}, \mathrm{q}, \\
\left.\mathrm{C}_{2} \mathrm{CH}_{3}\right) ; 1.03\left(3 \mathrm{H}, \mathrm{t}, \mathrm{CH}_{2} \mathrm{C}_{3}\right)\end{array}$ & 376 \\
\hline $4 a$ & 11.96 & 8.11 & 7.94 & 7.42 & 6.52 & 2.50 & 2.28 & $\begin{array}{l}3.40- \\
3.44\end{array}$ & 3.20 & $\begin{array}{l}\text { 2.80-2.96 }(1 \mathrm{H}, \mathrm{m}, \mathrm{CH}-\mathrm{NH}) ; \\
0.94-1,26\left(10 \mathrm{H}, \mathrm{m}, \mathrm{C}_{5} \underline{\mathrm{H}}_{10} \mathrm{CH}_{3}\right) ; \\
0.83\left(3 \mathrm{H}, \mathrm{t}, \mathrm{C}_{5} \mathrm{H}_{10} \mathrm{CH}_{3}\right)\end{array}$ & 370 \\
\hline $4 b$ & 11.98 & 8.11 & 7.85 & 7.41 & 6.51 & 2.49 & 2.31 & $\begin{array}{l}3.40- \\
3.44\end{array}$ & 3.22 & $\begin{array}{l}\text { 6.97-7.16 }\left(5 \mathrm{H}, \mathrm{m}, \mathrm{C}_{6} \mathrm{H}_{5}\right) ; 2.80- \\
2.93(1 \mathrm{H}, \mathrm{m}, \mathrm{CH}-\mathrm{N} \underline{\mathrm{H}}) ; 1.14(2 \mathrm{H}, \\
\left.\mathrm{d}, \mathrm{CH}_{2} \mathrm{C}_{6} \mathrm{H}_{5}\right)\end{array}$ & 364 \\
\hline 5 & 11.98 & 7.90 & 7.70 & 7.34 & 6.49 & 2.46 & 2.87 & $\begin{array}{l}3.60- \\
3.81\end{array}$ & 1.10 & $* *$ & 281 \\
\hline
\end{tabular}

* - 1.08-1.12 ppm multiplet has integrated intensity $9 \mathrm{H}$ and contains protons of $\mathrm{CH}_{3}$ groups of two ethyls and one propyl;

** - proton of the $\mathrm{OH}$ group is in deuterium exchange with water of the solvent.

$(2.63 \mathrm{~g})$ of allylsulfone 2 and 0.025 mole of $n$-hexylamine in $5 \mathrm{ml}$ of DMFA with stirring at $50-60^{\circ} \mathrm{C}$ for $6 \mathrm{~h}$. Add $50 \mathrm{ml}$ of water, and acidify the mixture with hydrochloric acid to $\mathrm{pH}=7$, then extract with ether. Evaporate the solvent; crystallize the precipitate with ethanol.

6-(2-Benzylaminopropylsulfonyl)-4-methyl-1,2-dihydroquinolin-2-one (4b) was prepared similarly to compound 4a using benzylamine instead of $n$-hexylamine.

Synthesis of 6-(2-hydroxypropylsulfonyl)-4-methyl1,2-dihydroquinolin-2-one (5). Dissolve 0.01 mole (2.63 g) of allylsulfone 2 in $5 \mathrm{ml}$ of DMFA when heating. Add 0.012 mole $(0.48 \mathrm{~g})$ of sodium hydroxide. Stir the mixture for $30 \mathrm{~min}$ at $20^{\circ} \mathrm{C}$, acidify with hydrochloric acid to $\mathrm{pH}=5-7$ and dilute with water. Filter the precipitate formed. Crystallize the product with $50 \%$ ethanol adding 1-2 drops of diluted hydrochloric acid.

\section{CONCLUSIONS}

1. The preparative method of alkylation of 4-methyl-2-oxo-1,2-dihydroquinoline-6-sulfinic acid with allyl bromide has been developed and 6-allylsulfonyl4-methyl-1,2-dihydroquinoline-2-one has been synthesized.

2. To obtain new biologically active substances in the range of sulfonyl derivatives of quinolone- 2 the conditions of the reactions of 6-allylsulfonyl-4-methyl-1,2dihydroquinoline-2-one with some of C-, $\mathrm{N}$ - and O-nucleophilic reagents have been investigated and the corresponding products of addition to the allyl fragment have been obtained.

3. The chemical transformations described can be considered as a convenient way to modify and diversify sulfonyl derivatives of 1,2-dihydroquinoline-2-one.

\section{REFERENCES}

1. Зубков В.О., Гриценко І.С., Цапко Т.О. // ЖОФХ. - 2009. - Т. 7, вип. 3 (27). - С. 30-34.

2. Зубков В.А., Цапко Т.А., Гриценко И.С., Малоштан Л.Н. // ИзвАН. Серия хим. - 2010. - №12. - С. 2272-2275.

3. Получение и свойства органических соединений серы / Под ред. Л.И.Беленького. - М.: Химия, 1998. $560 \mathrm{c.}$

4. Прилежаева Е.Н. // Успехи химии. - 2000. - T. 69, №5. - C. 403-446.

5. Amewu R., Gibbons P., Mukhtar A., Stachulski A.V. et al. // Org. Biomolec. Chem. - 2010. - №8 (9). - P. 2068-2077.

6. Cohen A., Crozet M.D., Rathelot P. // Molecules. - 2012. - Vol. 21, №18 (1). - P. 97-113.

7. Oja S., Saransaari P. // Proc. West. Pharmacol. Soc. - 2007. - Vol. 50. - P. 8-15.

8. Padmavathi V., Thriveni P., Sudhakar Reddy G., Deepti D. // Eur. J. Med. Chem. - 2008. - Vol. 43, №5. P. 917-924.

9. Samuele A., Kataropoulou A., Viola M., Zanoli S. et al. // Antiviral Res. - 2009. - Vol. 81, №1. - P. 47-55.

10. Shaaban M.R., Saleh T.S., Mayhoub A.S., Mansour A. et al. // Bioorg. Med. Chem. - 2008. - Vol. 16, №12. P. 6344-6352.

11. Silvestri R., De Martino G., La Regina G. et al. // J. Med. Chem. - 2005. - Vol. 46, №12. - P. 2482-2493. 
12. Tozkoparan B., Küpeli E., Yeşilada E., Ertan M. // Bioorg. Med. Chem. - 2007. - Vol. 15, №4. - P. 1808-1814.

13. Verbist B., Cleyn M., Surkyn M., Fraiponts E. et al. // Bioorg. Med. Chem. Lett. - 2008. - Vol. 18, №8. P. 2574-2579.

\section{ХІМІЧНІ ПЕРЕТВОРЕННЯ НА ОСНОВІ 6-АЛІЛСУЛЬФОНІЛ-4-МЕТИЛ-1,2-ДИГІДРОХІНОЛІН- 2-ОНУ 3 МЕТОЮ ПОШУКУ НОВИХ БІОЛОГІЧНО АКТИВНИХ РЕЧОВИН \\ T.0.Цапко}

Ключові слова: 1,2-дигідрохінолін-2-он; алілсульфрон; гетероциклічні сульфрони;

нуклеофільне приєднання

3 метою пошуку нових біологічно активних речовин серед сульфронільних похідних хінолону-2 нами було розроблено методику одержання зручного інтермедіату - 6-алілсульфоніл4-метил-1,2-дигідрохінолін-2-ону. Синтез здійснено шляхом алкілування вихідної 4-метил-2оксо-1,2-дигідрохінолін-6-сульфрінової кислоти алілбромідом у середовищі ацетонітрилу за присутності поташу з виходом цільового продукту 73\%. Наявність алільного фррагменту в одержаному 6-алілсульфоніл-4-метил-1,2-дигідрохінолін-2-оні дозволила провести реакції приєднання деяких C-, N- та О-нуклеофрілів. Незважаючи на сильний електроноакцепторний вплив сульфоонільної групи приєднання по подвійному зв'язку алілсульфрону відбувається за правилом Марковнікова. Взаємодію 6-алілсульфоніл-4-метил-1,2-диаідрохінолін-2-ону з метиленактивними сполуками проводили на прикладі таких речовин як діетиловий естер малонової кислоти та етиловий естер ціанооцтової кислоти при кип'ятінні в абсолютному етанолі в присутності натрію етилату, отримавши етилові естери 2-R-3-метил-4(4-метил-2-оксо-1,2-дигідрохінолін-6-сульфооніл)бутанової кислоти з виходами 73-79\%. При дії на 6-алілсульфоніл-4-метил-1,2-дигідрохінолін-2-он N-нуклеофрільних реагентів, зокрема первинних аліфратичних амінів, утворюються 6-(2-алкіламінопропілсульфоніл)-4-метил-1,2дигідрохінолін-2-они. Дана реакція перебігає при нагріванні у диметилформаміді до 50-60 $\mathrm{C}$ протягом 6 год, виходи продуктів складають 35-40\%. Гідратація алільного фррагменту при дії натрію гідроксиду перебігала в тому самому розчиннику при $20^{\circ} \mathrm{C}$ з утворенням 6-(2-гідроксипропілсульфоніл)-4-метил-1,2-дигідрохінолін-2-ону з виходом 62\%. Зазначені напрямки модифрікації відкривають перспективу для створення широких рядів нових сполук з різноманітними фрункціональними групами в алкілсульфронільному фррагменті хінолонів. Такий підхід також дозволяє впливати на такі властивості нових БАВ, як молекулярна маса, ліпофрільність, кислотність тощо, що має важливе значення для проникності речовин крізь біологічні мембрани.

\section{ХИМИЧЕСКИЕ ПРЕВРАЩЕНИЯ НА ОСНОВЕ 6-АЛЛИЛСУЛЬФОНИЛ-4-МЕТИЛ-1,2- ДИГИДРОХИНОЛИН-2-ОНА С ЦЕЛЬЮ ПОИСКА НОВЫХ БИОЛОГИЧЕСКИ АКТИВНЫХ ВЕЩЕСТВ \\ Т.А.Цапко}

Ключевые слова: 1,2-дигидрохинолин-2-он; аллилсульфрон; гетероциклические сульфроны; нуклеофильное присоединение

Для поиска новых биологически активных веществ среди сульфонильных производных хинолона-2 нами была разработана методика получения удобного интермедиата - 6-аллилсульфонил-4-метил-1,2-дигидрохинолин-2-она. Синтез осуществлен путем алкилирования исходной 4-метил-2-оксо-1,2-дигидрохинолин-6-сульфриновой кислоты аллилбромидом в среде ацетонитрила и в присутствии поташа с выходом целевого продукта $73 \%$. Наличие аллильного фррагмента в полученном 6-аллилсульфонил-4-метил-1,2-дигидрохинолин-2-оне позволило провести реакции присоединения некоторых C-, N- и О-нуклеофилов. Несмотря на сильное электроноакцепторное влияние сульфоннильный группы присоединение по двойной связи алилсульфрона происходит по правилу Марковникова. Взаимодействие 6-аллилсульфонил4-метил-1,2-дигидрохинолин-2-она с метиленактивными соединениями проводили на примере таких веществ, как диэтиловый эфир малоновой кислоты и этиловый эфир цианоуксусной кислоты при кипячении в абсолютном этаноле в присутствии натрия этилата, получив этиловые эфриры 2-R-3-метил-4-(4-метил-2-оксо-1,2-дигидрохинолин-6-сульфронил) бутановой кислоты с выходами 73-79\%. При действии на 6-аллилсульфонил-4-метил-1,2дигидрохинолин-2-он N-нуклеофильных реагентов, в частности первичных алифратических аминов, образуются 6-(2-алкиламинопропилсульфонил)-4-метил-1,2-дигидрохинолин-2-оны. Данная реакция проходит при нагревании в диметилформамиде до 50-60 ${ }^{\circ} \mathrm{C}$ в течение 6 часов, выходы продуктов составляют 35-40\%. Гидратация алильного фррагмента при действии натрия гидроксида протекала в том же растворителе при $20^{\circ} \mathrm{C}$ с образованием 6-(2-гидроксипропилсульфронил)-4-метил-1,2-дигидрохинолин-2-она с выходом 62\%. Указанные направления модифрикации открывают перспективу для создания широких рядов новых соединений с различными функциональными группами в алкилсульфонильном фррагменте хинолонов. Данный подход также позволяет влиять на такие свойства новых БАВ, как молекулярная масса, липофильность, кислотность и т. д., что имеет важное значение для проницаемости веществ через биологические мембраны. 